\title{
Effect of multimorbidity on quality of life in adult with cardiovascular disease: a cross- sectional study
}

\author{
Bijan Shad ${ }^{1}$, Asieh Ashouri ${ }^{1,2}$, Tolou Hasandokht ${ }^{1,3^{*}} \mathbb{D}$, Fatemeh Rajati ${ }^{4}$, Arsalan Salari ${ }^{1}$, Moona Naghshbandi ${ }^{1}$ \\ and Fardin Mirbolouk
}

\begin{abstract}
Background: The aim of present study was to describe the effect of multimorbidity on Health-Related Quality of Life (HRQoL) in patients with coronary artery disease (CAD).

Methods: A cross-sectional study with a simple sampling method of 296 patients undergoing coronary artery bypass surgery in a referral hospital of the northern part of Iran was conducted between April, 2015 and September, 2016. Multimorbidity was defined as the presence of at least two chronic diseases based on self-reporting and medical records. HRQoL was measured using the 36-item short form (SF-36) health status survey. We used analysis of variance (ANOVA) to assess the effect of multimorbidity on mental and physical component of HRQoL.

Results: Approximately, 69\% of CAD patients had at least one other disease like diabetes or hypertension. Patients without multimorbidity compared with patients with multimorbidity were significantly older $(p=0.012)$ and more educated $(p=0.002)$. Both physical and mental component score of HRQoL was better in patients without any morbidity (48.82 vs. 43.93 with $95 \% \mathrm{Cl}$ of mean difference: $3.37-6.42$ and 54.85 vs. 50.44 with $95 \% \mathrm{Cl}$ of mean difference: 1.68-7.15, respectively). Both physical and mental component score was significantly lower in female and lower educated patients (physical mean score 43.07 vs. 46.54 with $P=.001$ and 42.53 vs. 46.82 with $P<.001$ and mental mean score 49.98 vs. 52.65 with $P=.055$ and 49.80 vs. 52.75 with $P=.022$ for sex and education, respectively). Also, two-way ANOVA showed that regards to morbidity, physical component score was greater in patients with lower education level than higher education level $(P<.001)$.
\end{abstract}

Conclusion: The findings of this study suggest that women, lower education level and overweight patients reported lower quality of life. HRQoL is affected by multimorbidity among CAD patients specially in less educated.

Keywords: Multimorbidity, Quality of life, Comorbidity, Coronary artery disease

\section{Background}

Coronary artery bypass graft (CABG) surgery is known as an effective treatment for advanced coronary artery disease (CAD) with high survival rate in the early years of surgery [1]. Evidence showed that CABG surgery can reduce symptoms and disability [2]. Patients with CAD

\footnotetext{
* Correspondence: tolou.hasandokht@gmail.com;

Tolou.hassandokht@gums.ac.ir

'Department of Cardiology, Cardiovascular Diseases Research Center, Heshmat Hospital, School of Medicine, Guilan University of Medical Sciences, Rasht, Iran

${ }^{3}$ Department of Community Medicine, School of Medicine, Guilan University of Medical Sciences, Rasht, Iran

Full list of author information is available at the end of the article
}

needs continuous care and requires multiple risk factor like diabetes (DM), hypertension (HTN), and hyperlipidemia management strategies. Coexistence of two or more chronic conditions in one patient was defined as multimorbidity (MM) [3], which have negative effect on physical and mental function and higher need to major treatment [4]. According to world health organization definition of health, quality of life must be considered as a substantial health outcome in every disease management. Health-Related Quality of Life (HRQoL) measurement provides an acceptable and valid method for assessing the impact of disease on patients' function, activity and well-being [5]. Previous studies showed a 
lower quality of life among subjects with CAD $[6,7]$ and also when compared with the general population [8]. CABG, is a known treatment for CAD, could improve health-related quality of life (HRQoL), and increase survival $[8,9]$. However, recent studies discuss about some factors could change the quality of life after CABG $[10,11]$. Luc Noyez et al. in a review article mentioned about considering demographic factors and comorbidity in evaluation of HRQoL after cardiac surgery [10]. Several factors such smoking, high alcohol intake, low socioeconomic status, presence of other disease, low education level, and overweight were found to affect improvement of quality of life after CABG [12, 13]. Moreover, impaired HRQoL in patients with MM has been described previously $[14,15]$. Studies about the impact of multimorbidity on HRQoL after cardiac surgery is limited [16]. Knowing the HRQoL in CAD patients with MM could help clinicians to design and development of appropriate health programs.

The aim of this study was to describe the effect of multimorbidity on HRQoL in patients 5 years post CABG.

\section{Methods}

\section{Study design}

This is a cross sectional study evaluating the health status of CAD patients after 5 years CABG. Health survey conducted between 2015 and 2016 in a teaching hospital located in the northern part of Iran.

\section{Data collection}

A simple sampling of patients older than 20 years underwent CABG in 2010 who were alive on discharge time were drawn from hospital records $(n=354)$. Patients were invited to participate in the study through telephone call. A specific visit time was scheduled for all participants. Those patients who refused to participation after two times invitation were excluded from the study $(n=7)$.
Other reasons for attrition include death during 5 years (15), wrong registered phone number (32), home transition (4) (Fig. 1).

Subjects were requested to bring all medications currently taken together with their medical reports.

On the day of the study visit, after obtaining informed consent, structured interview were performed by a trained research assistant. Questions were about demographic characteristics including age, gender, living location (rural or city), education level (less than 12 years of education or higher) and smoking status. Data on any recent cardiac symptoms like chest pain and exertional dyspnea post surgery were assessed by a cardiologist. Cardiac symptoms were presented as yes or no. Anthropometric parameters like height and weight were measured in light clothes without shoes by a precalibrated digital SECA scale and portable stadiometer by a research assistant. We calculated body mass index (BMI) for study population as weight in $\mathrm{kg}$ divided by squared height in $\mathrm{m}$. Normal weight was defined as BMI $<25$, overweight as BMI 2529.9 , and obesity as $B M I \geq 30 \mathrm{~kg} / \mathrm{m}^{2}$. Medical history including diabetes and hypertension was considered present according to patients self report and medications. We categorized multimorbidity to 1 ) CAD without any morbidity $(n=91), 2)$ CAD plus diabetes $(n=$ $30)$, 3) CAD plus hypertension $(n=76), 4)$ CAD plus diabetes and hypertension $(n=99)$.

All subjects were interviewed to assess HRQoL by 36-item short form (SF-36) health status survey. The SF-36 questionnaire measured daily functioning abstracted into two summary measures: physical component summary (PCS) and mental component summary (MCS) scales [17]. PCS scale included four domains including physical functioning, role physical, bodily pain, general health. MCS scale consist of vitality, social functioning, role emotional, and mental health. Total score range for each item was varied from 0 to 100 scales from worst to best. Persian version of questionnaire was translated and validated by Montazeri et al. [18]. A

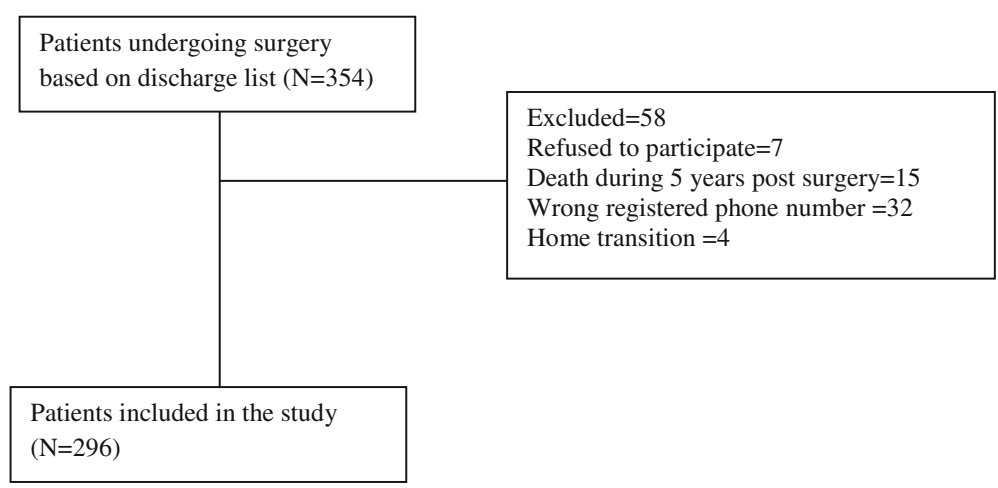

Fig. 1 A chart reporting the flow of participants through each stage of the study 
minimum difference in three to five points is considered clinically important based on a previous review [19].

\section{Statistical analysis}

Mean (SD, range) and frequency (percentage) was reported to describe the study population. Normal distribution assumption was checked by skewness and Kurtosis criteria (lower than 1) and Kolmogrov-Smirnov test. Independent sample t-test and Chi-square test was performed to compare continuous and categorical variables, respectively. Pearson correlation was used to estimate the correlation between variables. Mean score of physical and mental component were obtained for patients with and without morbidity and groups according to multimorbidity (without morbidity, with diabetes only, with hypertension only and with comorbidity of diabetes and hypertension). One-way analysis of variance (ANOVA) was performed to compare physical or mental component score between groups and Tukey HSD test was used for post hoc pair comparisons. In the nonhomogeneity of variances condition, Welch $\mathrm{F}$ ratio statistics and Games-Howell post hoc test was used to test the difference of physical or mental component score between comorbidity groups. Two-way ANOVA were performed to compare component of quality of life score between co-morbidity groups adjusted for demographic variables. Data were analyzed by SPSS software version 18 and $P<0.05$ was considered statistically significant.

\section{Results}

Patients' characteristics are shown in Table 1. In total, 296 patients aged between 44 and 89 years (mean age $62.5 \pm 8.1$ ) were included in the study. Two hundred and one $(68 \%)$ were male. Ninety one patients $(31 \%)$ had no comorbid chronic conditions. Compared with CAD patients without multimorbidity, those with multimorbidity were younger (mean age 64.03vs. 62.90 years), more female and more with lower education level.

Descriptive statistics of quality of life scores are presented in Table 2. Both physical and mental component score of quality of life was significantly lower in patients with multimorbidity compared with those without (48.82

Table 1 Patients characteristics in total and by co-morbidities with CAD groups

\begin{tabular}{|c|c|c|c|c|c|c|c|c|}
\hline & \multirow[b]{2}{*}{$\begin{array}{l}\text { Total } \\
(n=296)\end{array}$} & \multirow{2}{*}{$\begin{array}{l}\text { Without } \\
\text { co-morbidity } \\
(n=91,30.7 \%)\end{array}$} & \multicolumn{4}{|c|}{ With co-morbidity } & \multirow[b]{2}{*}{$P^{*}$} & \multirow[b]{2}{*}{$p^{* *}$} \\
\hline & & & $\begin{array}{l}\text { Total } \\
(n=205,69.2 \%)\end{array}$ & $\begin{array}{l}\text { DM only } \\
(n=30,10.1 \%)\end{array}$ & $\begin{array}{l}\text { HTN only } \\
(n=76,25.6 \%)\end{array}$ & $\begin{array}{l}\mathrm{DM}+\mathrm{HTN} \\
(n=99,33.4 \%)\end{array}$ & & \\
\hline Sex, no. (\%) & & & & & & & $<.001$ & $<.001$ \\
\hline Male & $201(68)$ & 78(39) & $123(61)$ & $25(12)$ & $52(26)$ & $46(23)$ & & \\
\hline Female & $95(32)$ & $13(14)$ & $82(86)$ & $5(5)$ & $24(25)$ & $53(56)$ & & \\
\hline Living location, no. (\%) & & & & & & & .419 & .224 \\
\hline Urban & $238(80)$ & $77(32)$ & $161(68)$ & $23(10)$ & $57(24)$ & $81(34)$ & & \\
\hline Rural & $58(20)$ & $14(24)$ & $44(76)$ & $7(12)$ & 19(33) & $18(31)$ & & \\
\hline Education, no. (\%) & & & & & & & .001 & .002 \\
\hline$<$ diploma & $96(32)$ & 18(19) & $78(81)$ & $5(5)$ & $30(31)$ & $43(45)$ & & \\
\hline$>=$ diploma & $200(68)$ & $73(37)$ & $127(63)$ & $25(13)$ & $46(23)$ & $56(28)$ & & \\
\hline Symptom, no. (\%) & & & & & & & .943 & .559 \\
\hline Yes & $97(33)$ & $32(33)$ & $65(67)$ & $10(10)$ & $24(25)$ & $31(32)$ & & \\
\hline No & 199(67) & $59(30)$ & $140(70)$ & $20(10)$ & $52(26)$ & $68(34)$ & & \\
\hline Smoking, no. (\%) & & & & & & & $<.001$ & $<.001$ \\
\hline Yes & $83(28)$ & $36(43.3)$ & $47(56.6)$ & $8(9.6)$ & $23(28)$ & 16(19) & & \\
\hline No & 213(72) & $55(28.5)$ & $158(74)$ & $22(10)$ & $53(25)$ & 83(39) & & \\
\hline $\mathrm{BMI}{ }^{* * *}$, mean $(\mathrm{SD})$ & $28.37(4.70)$ & $26.17(3.58)$ & $29.32(4.82)$ & $28.55(2.45)$ & $28.41(4.88)$ & $30.23(5.12)$ & $<.001$ & $<.001$ \\
\hline Normal & $76(26)$ & $36(47)$ & $40(53)$ & $2(3)$ & $18(24)$ & $20(26)$ & & \\
\hline Overweight & $118(41)$ & $40(34)$ & $78(66)$ & $22(19)$ & $26(22)$ & $30(25)$ & & \\
\hline Obese & $95(33)$ & $11(12)$ & $84(88)$ & $3(3)$ & $32(34)$ & $49(51)$ & & \\
\hline Age, mean (SD) & $62.52(8.10)$ & $64.03(9.45)$ & 63.33(8.36) & $62.85(5.48)$ & $60.03(7.21)$ & $62.90(8.14)$ & .065 & .012 \\
\hline
\end{tabular}

*P-value was reported for comparison between four co-morbidity groups (without co-morbidity, DM, HTN, DM+HTN)

**P-value was reported for comparison between patients with and without co-morbidity

***BMl data was missing for $7(2 \%)$ of patients 
Table 2 Physical and mental component score in total and by comorbidities with CAD groups

\begin{tabular}{|c|c|c|c|c|c|c|}
\hline & Total $(n=296)$ & None $(n=91)$ & DM only $(n=30)$ & HTN only $(n=76)$ & $\mathrm{DM}+\mathrm{HTN}(n=99)$ & $P$ \\
\hline Physical component & & & & & & $<.001^{*}$ \\
\hline Mean (SD) & $45.43(7.84)$ & $48.82(4.81)$ & $41.37(10.56)$ & 45.65(7.39) & $43.37(8.30)$ & \\
\hline Min-max & $23.54-60.00$ & $36.10-57.17$ & $23.54-54.11$ & $25.88-56.31$ & $26.83-60.00$ & \\
\hline Mental component & & & & & & $.018^{* *}$ \\
\hline Mean (SD) & $51.80(11.21)$ & $54.85(10.82)$ & $50.23(12.17)$ & 49.99(11.48) & $50.84(10.65)$ & \\
\hline Min-max & 29.29-74.53 & 29.29-74.53 & $31.59-68.32$ & $33.34-73.34$ & $31.50-70.65$ & \\
\hline
\end{tabular}

$P$-value was reported for comparison between patients with and without comorbidity

*There was a significant difference between none co-morbidity group and DM $(P=.004)$, HTN $(P=.009)$ and DM $+\mathrm{HTN}(P<.001)$ based on Games-Howell post hoc test **There was a significant difference between none co-morbidity group and HTN $(P=.026)$ based on Tukey HSD post hoc test

vs. 43.93 with $95 \% \mathrm{CI}$ of mean difference: $3.37-6.42$ and 54.85 vs. 50.44 with $95 \% \mathrm{CI}$ of mean difference: 1.68 7.15 for physical and mental component score, respectively) (table 2). There was a weak relation between physical and mental component score $(r=.206, P<.001)$.

Physical component score comparison between different multimorbidity groups showed that there was a significant difference between non multimorbidity group and each of DM $(P=.004)$, HTN $(P=.009)$ and DM + HTN $(P<.001)$ groups. But there was no any differences between DM, HTN and DM + HTN pairs groups $(P>.20$ for all other comparisons, based on Games-Howell post hoc test). Also, pairwise comparison of mental component score between groups showed that there was only a significant difference between CAD group without morbidity and those with HTN only group $(P=.026)$. There were no other significant differences between DM, HTN and DM + HTN pairs groups $(P>.05$ for all other comparison based on Tukey post hoc test).

Assessing relationships between demographic characteristics and physical and mental component score didn't showed any significant association; for age $(r=-.07$ with $P=.241$ and $r=-.03$ with $P=.636$, respectively), living location $(P=.796$ and $P=.123$, respectively), smoking status $(P=.716$ and $P=.880$, respectively) and cardiac symptom $(P=.452$ and $P=.535$, respectively). But, Physical component score was related to the patients sex $(P=.001)$, educational level $(P<.001)$ and BMI $(P=.044)$.

Women reported statistically significant lower PCS than men (mean score 43.07 vs. $46.54,95 \%$ CI of mean difference: $1.40-5.54, \mathrm{P}=.001$ ). Also, physical component score in patients with higher educational level was grater than those with low education level (mean score 46.82 vs $42.53,95 \%$ CI of mean difference: 2.17-6.41, $P<.001)$. Overweight and obese patients reported lower PCS score when compared to normal weight. The mean score of PCS in normal, overweight and obesity were $48.23,45.91$, and 42.86 respectively, $\mathrm{P}=.044$ ).

Post hoc test showed patients with obesity had lower physical component score compared with normal or overweight patients $(95 \% \mathrm{CI}$ of mean difference: 2.94-7.81 with $P<.001$ and $95 \% \mathrm{CI}$ of mean difference: $.48-5.63$ with $P=.015$, respectively). There was no significant difference between normal and overweight patients regard to physical component score $(95 \% \mathrm{CI}$ of mean difference: $-.05-4.69$ with $P=.057$ ).

Mental component score was related to the patients sex $(P=.052)$ and educational level $(P=.022)$ but not related to the BMI $(P=.918)$. Mental component score was significantly lower in female (mean score 49.98 vs. $52.65,95 \% \mathrm{CI}$ of mean difference: $-.06-5.41, \mathrm{P}=.052$ ), and in patients with lower education level (mean score 49.80 vs. $52.75,95 \% \mathrm{CI}$ of mean difference: .44-5.47, $\mathrm{P}=.022$ ).

Regards to both physical and mental component score, there were no interaction between patients with and without multimorbidity groups and sex $(P=.470$ and $P=.791$, respectively) or BMI level $(P=.894$ and $P=.163$, respectively). It means that there was no significant difference in the HRQoL score among patients with multimorbidity between sex or BMI category (Fig. 2).

For mental component score, there was no interaction between with and without multimorbidity groups and education level $(P=.633)$. However for physical component score, two-way ANOVA showed an interaction between patients with and without multimorbidity group and educational level $(P<0.001$, Fig. 1$)$. So, in patients with lower education level, decrease in the physical component score was greater and clinically significant (mean score 40.54 vs. $51.18,95 \%$ CI of mean difference: 7.39 13.91, $p<0.05)$, but in patients with higher education level, decreasing the physical component score was low and not clinical significant (mean score 46.01 vs. 48.24, 95\%CI of mean difference: .59-3.87, $p<0.05$ ) (Fig. 1).

\section{Discussion}

The result of our study showed that $69 \%$ of study population had at least one morbidity condition along with CAD. The most common morbidity was hypertension among CAD patients. Hypertension is a one of the important disease in patients with CAD [20]. In the present 


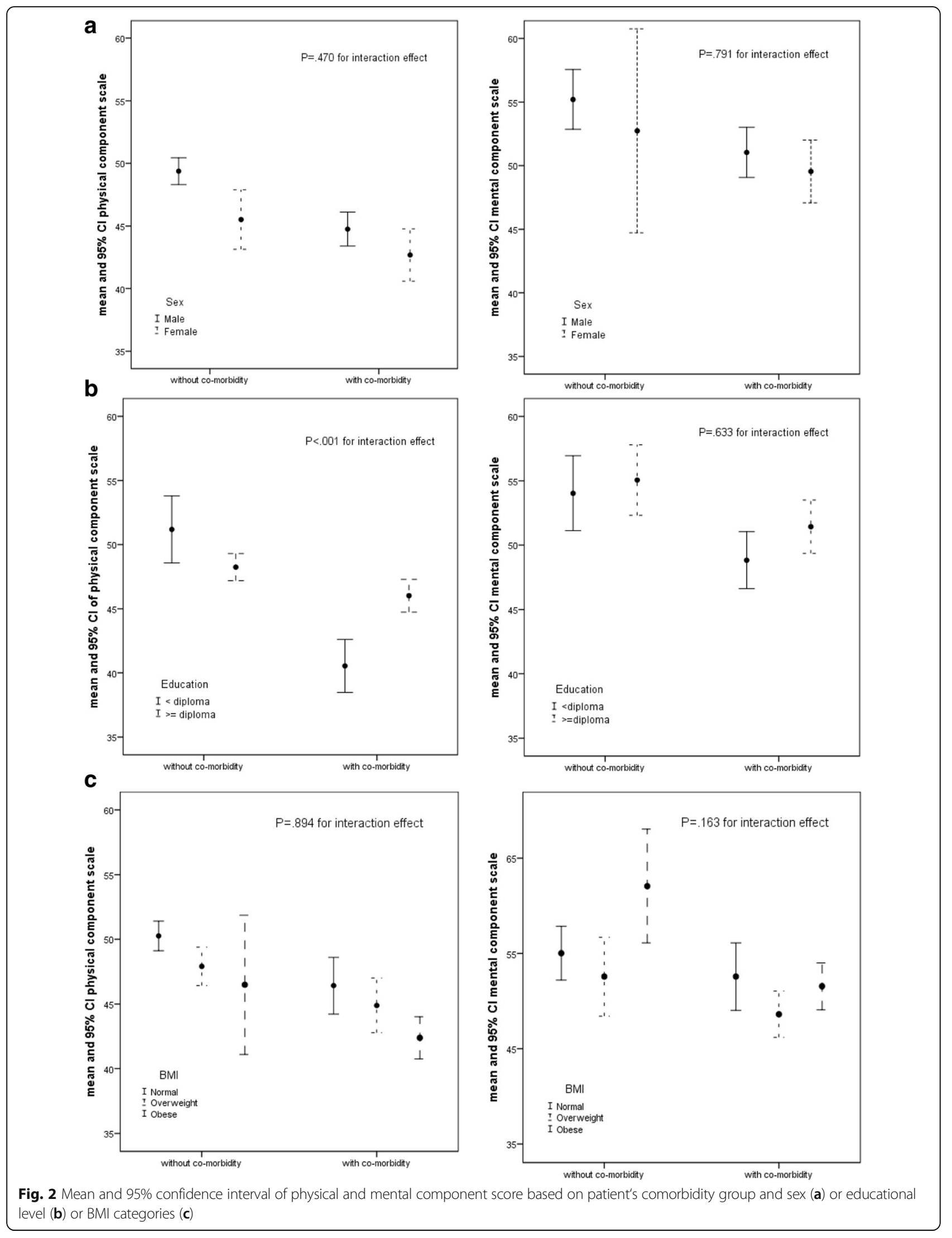


study, patients with multimorbidity were slightly older than those without multimorbidity. Patients with several risk factors were more at risk of coronary artery disease [21].According to a recent systematic review, prevalence of multimorbidity increase in older people [22]. And also parallel with previous studies, multimorbidity was more common among women $[3,23,24]$ and less educated people [25]. The prevalence of multimorbidity in our hospital base data was higher than general population in Ramezankhani's study [26]. In the recent study, about $35-40 \%$ of general population had multiple cardiovascular risk factors like hypertension or diabetes [26]. Although the high prevalence of multiple disease in patients admitted in hospitals is expected. But Agborsangaya and colleague [24] showed co occurrence of multiple disease is prevalent in 18 years and over in the general population. Hence, development and implantation of prevention program for general population seems to be important.

Physical and mental component of quality of life in CAD patients with multimorbidity were worse when compared to those without. Furthermore, in the present study CAD patients without any morbidity reported better PCS compared to CAD plus HTN, or plus DM or both. Also, we observed a reduction in the score of mental component of HRQoL among CAD group with hypertension when compared to CAD without any morbidities. Similarly, the literature showed cardiomethabolic disorder was associated with reduced score in physical component of HRQoL rather than mental component [27]. Interestingly, we didn't detect any dose response relationship between increasing in the number of morbidity and PCS as well as MCS. Although, a recent systematic review reported an association between number of multimorbidity and clinical outcome in patients with cardiovascular disease [16]. But, to the best of knowledge there is not any study focusing on such association with quality of life in cardiovascular disease. However, several studies showed the negative effect of increasing in the number of morbidity and HRQoL in diabetic patient $[28,29]$.

In our study, lower physical and mental component of HRQoL were observed in women and less educated CAD patients, consistent with previous studies $[5,30]$. We found in lower educated group, presence of multimorbidity have clinically important effect on PCS. While in higher educated patients, we didn't find any clinically effect of multimorbidity on PSC. which is confirmed by Yulian Zhanget al study [31]. This issue indicated the importance of patient's knowledge about lifestyle behavior and self management [32]. Similarly, Choowattanapakorn et al. in a multicenter study showed self-care behavior was a predictive factor of PCS in 345 diabetic patients. When patients more know about causes of disease and treatment, they can better modify their health behaviors. According to the previous evidence, women more express such symptoms related to energy and fatigue than do men [33]. On the other hands, Sylvie S. L et al. in a multicenter, prospective cohort study showed better social function score in men compared to women [34]. Moreover, in the present study patients with higher BMI level reported lower physical component of quality of life. But, we didn't observe such association regarding mental component. Similar findings have been previously reported in several studies $[35,36]$.

It is well-documented that CAD, DM and HTN is not only associated with increased morbidity and mortality but also with substantial impairment in HRQoL [37]. Martin Fortin et al. in a systematic review showed a negative effect of multimorbidity on QOL in primary care setting [14]. In recent times, patient health status is considered important factor both as a risk factor and a health outcome. The AHA's strategic goals is improvement in cardiovascular health By 2020 [38]. Therefore, comorbidities management programs along with cardiac symptoms management should be addressed improve health status and HR-QOL for CAD patients. Notable problem in managing patients with $\mathrm{MM}$ is a single disease view of clinical guidelines and protocols. Moreover, polypharmacy, multiple doctor appointment and engagement in several lifestyle modification were other issues can affect the patients' health status and HR-QOL.

Our study involved some limitations that should be considered. We had small number of subjects in diabetes subgroup, which decrease the statistical power. Furthermore, we didn't considered the comorbidity duration among study population, that could influence on HRQoL state. Additionally, the glycemic control, blood pressure level and depressive mood could have affect on HRQoL, which didn't assess in the present study. Although the location of data gathering was the referral cardiovascular hospital of our province, but majority of patients were related to rural area and lower socioeconomic region. Thus, we can not generalize our findings to total population.

\section{Conclusion}

This study indicated that more than $50 \%$ of CAD patients had at least one other morbidity like hypertension or diabetes. Patients with multimorbidity were younger, more often female and less educated. CAD patients with one or more other morbidities reported significantly lower PCS physical of HRQoL compared to those without any morbidities. But, regard to mental component, CAD patients with HTN had the worse score than those without any morbidity. Patients presence of multimorbidity in less educated patients cause more clinically effect on PCS compared to MCS. 


\section{Abbreviations}

ANOVA: analysis of variance; CABG: Coronary artery bypass surgery; CAD: Coronary Artery Disease; DM: Diabetes; HRQoL: Health-Related Quality of Life; HTN: Hypertension; MCS: Mental component summary; MM: Multimorbidity; PCS: Physical component summary; SF-36: 36-item short form

\section{Acknowledgements}

The authors thank research chancellor of Guilan University of medical science for supporting the study.

\section{Funding}

Vice-chancellor for research of Guilan University of medical science according to Helsinki declaration (research number: 94,070,709).

\section{Availability of data and materials}

The datasets used and/or analyzed during the current study available from the corresponding author on reasonable request.

\section{Authors' contributions}

TH was the main investigator, who designed the study, analyzed the data and wrote the paper. AS contributed to the study design, and writing of the paper. AA contributed to analysis of the data and writing the result of manuscript. FR contributed to analysis of data and helped in writing. BSH contributed to interpretation of the data and writing of manuscript. MN helped in study design andwriting the manuscript. FM contributed in interpretation of data and revised the final draft. All authors read and approved the final manuscript.

\section{Ethics approval and consent to participate}

Study design and patient informed consent was approved by vice-chancellor for research of Guilan University of medical science according to Helsinki declaration. All participants sing informed consent.

\section{Consent for publication}

Not applicable

\section{Competing interests}

The authors declare that they have no competing interests.

\section{Publisher's Note}

Springer Nature remains neutral with regard to jurisdictional claims in published maps and institutional affiliations.

\section{Author details}

'Department of Cardiology, Cardiovascular Diseases Research Center, Heshmat Hospital, School of Medicine, Guilan University of Medical Sciences, Rasht, Iran. ${ }^{2}$ School of Health, Guilan University of Medical Sciences, Rasht, Iran. ${ }^{3}$ Department of Community Medicine, School of Medicine, Guilan University of Medical Sciences, Rasht, Iran. ${ }^{4}$ Departments of health education and promotion, School of Public Health, Kermanshah University of Medical Sciences, Kermanshah, Iran.

Received: 13 May 2017 Accepted: 1 December 2017

Published online: 08 December 2017

\section{References}

1. Carr BM, et al. Long-term post-CABG survival: performance of clinical risk models versus actuarial predictions. J Card Surg. 2016;31(1):23-30.

2. Lindsay $G M$, et al. Coronary artery disease patients' perception of their health and expectations of benefit following coronary artery bypass grafting. J Adv Nurs. 2000;32(6):1412-21.

3. Loza E, et al. Multimorbidity: prevalence, effect on quality of life and daily functioning, and variation of this effect when one condition is a rheumatic disease. Seminars in arthritis and rheumatism. Elsevier; 2009;38:312-19. doi: 10.1016/j.semarthrit.2008.01.004

4. Smith SM, et al. Managing patients with multimorbidity: systematic review of interventions in primary care and community settings. BMJ. 2012;345: e32190. doi:10.1136/bmj.e5205.
5. De Smedt D, et al. Health related quality of life in coronary patients and its association with their cardiovascular risk profile: results from the EUROASPIRE III survey. Int J Cardiol. 2013;168(2):898-903.

6. Xie J, et al. Patient-reported health status in coronary heart disease in the United States age, sex, racial, and ethnic differences. Circulation. 2008:118(5):491-7.

7. Loponen $\mathrm{P}$, et al. HRQoL after coronary artery bypass grafting and percutaneous coronary intervention for stable angina. Scand Cardiovasc J. 2009:43(2):94-9.

8. Samano N, et al. Graft patency is associated with higher health-related quality of life after coronary artery bypass surgeryt. Interact Cardiovasc Thorac Surg. 2017;24(3):388-94.

9. McKenzie LH, Simpson J, Stewart M. A systematic review of pre-operative predictors of post-operative depression and anxiety in individuals who have undergone coronary artery bypass graft surgery. Psychol Health Med. 2010;15(1):74-93.

10. Noyez L, de Jager MJ, Markou ALP. Quality of life after cardiac surgery: underresearched research. Interact Cardiovasc Thorac Surg. 2011:13(5): $511-5$.

11. Middel B, et al. Decline in health-related quality of life 6 months after coronary artery bypass graft surgery: the influence of anxiety, depression, and personality traits. J Cardiovasc Nurs. 2014;29(6):544-54.

12. Lindsay $\mathrm{GM}$, et al. Assessment of changes in general health status using the short-form 36 questionnaire 1 year following coronary artery bypass grafting. Eur J Cardiothorac Surg. 2000;18(5):557-64.

13. Welke KF, et al. Patient characteristics can predict improvement in functional health after elective coronary artery bypass grafting. Ann Thorac Surg. 2003;75(6):1849-55

14. Fortin $\mathrm{M}$, et al. Multimorbidity and quality of life in primary care: a systematic review. Health Qual Life Outcomes. 2004;2(1):51.

15. Katsi, V., et al., [PP. 05.17] diabetic hypertensives and quality of life. J Hypertens, 2016. 34: p. e144-e145.

16. Tisminetzky M, Goldberg R, Gurwitz JH. Magnitude and impact of multimorbidity on clinical outcomes in older adults with cardiovascular disease: a literature review. Clin Geriatr Med. 2016;32. doi:10.1371/journal. pone.0169426

17. Ware, J.E., M. Kosinski, and S. Keller, SF-36 physical and mental health summary scales: a user's manual. 1994: Health Assessment Lab.

18. Montazeri A, et al. The short form health survey (SF-36): translation and validation study of the Iranian version. Qual Life Res. 2005;14(3):875-82.

19. Farivar SS, Liu H, Hays RD. Half standard deviation estimate of the minimally important difference in HRQOL scores? Expert Rev Pharmacoecon Outcomes Res. 2004;4(5):515-23.

20. Tisminetzky $M$, et al. Multiple chronic conditions and psychosocial limitations in patients hospitalized with an acute coronary syndrome. Am J Med. 2001;129(6):608-14.

21. Mamudu HM, et al. The effects of multiple coronary artery disease risk factors on subclinical atherosclerosis in a rural population in the United States. Prev Med. 2016;88:140-6.

22. Marengoni $\mathrm{A}$, et al. Aging with multimorbidity: a systematic review of the literature. Ageing Res Rev. 2011;10(4):430-9.

23. Fortin $\mathrm{M}$, et al. Prevalence estimates of multimorbidity: a comparative study of two sources. BMC Health Serv Res. 2010;10:193-214

24. Agborsangaya $\mathrm{CB}$, et al. Multimorbidity prevalence and patterns across socioeconomic determinants: a cross-sectional survey. BMC Public Health. 2012;12(1):201.

25. Larsen FB, et al. A latent class analysis of multimorbidity and the relationship to socio-demographic factors and health-related quality of life. A National Population-Based Study of 162,283 Danish adults. PLoS One. 2017;12(1):e0169426

26. Ramezankhani A, et al. Sex-specific clustering of metabolic risk factors and their association with incident cardiovascular diseases: a population-based prospective study. Atherosclerosis. 2017;263(Supplement C):249-56.

27. Walker $V$, et al. Effect of multimorbidity on health-related quality of life in adults aged 55 years or older: results from the SU.VI.MAX 2 cohort. PLoS One. 2016;11(12):e0169282.

28. Ose $\mathrm{D}$, et al. Health related quality of life and comorbidity. A descriptive analysis comparing EQ-5D dimensions of patients in the German disease management program for type 2 diabetes and patients in routine care. BMC Health Serv Res. 2011;11(1):179.

29. Adriaanse $M C$, et al. The impact of comorbid chronic conditions on quality of life in type 2 diabetes patients. Qual Life Res. 2016;25(1):175-82. 
30. Uchmanowicz I, et al. Influence of diabetes on health-related quality of life results in patients with acute coronary syndrome treated with coronary angioplasty. Acta Diabetol. 2013;50(2):217-25.

31. Zhang $Y$, et al. Health-related quality of life and its influencing factors for patients with hypertension: evidence from the urban and rural areas of Shaanxi Province, China. BMC Health Serv Res. 2016;16(1):277.

32. Hasandokht $\mathrm{T}$, et al. Lifestyle interventions for hypertension treatment among Iranian women in primary health-care settings: results of a randomized controlled trial. J Res Med Sci. 2015;20(1):54.

33. Bengtsson C, et al. Prevalence of subjectively experienced symptoms in a population sample of women with special reference to women with arterial hypertension. Scand J Prim Health Care. 1987;5(3):155-62.

34. Leung Yinko SSL, et al. Health-related quality of life in premature acute coronary syndrome: does patient sex or gender really matter? I Am Heart Assoc. 2014:3(4):e000901. doi:10.1161/jaha.114.000901.

35. Jia H, Lubetkin El. The impact of obesity on health-related quality-of-life in the general adult US population. J Public Health. 2005;27(2):156-64

36. Lopez-Garcia E, et al. Metabolically healthy obesity and health-related quality of life: a prospective cohort study. Clin Nutr. 2017;36(3):853-60

37. Yinko SSL, et al. Health-related quality of life in premature acute coronary syndrome: does patient sex or gender really matter? J Am Heart Assoc. 2014:3(4):e000901.

38. Rumsfeld JS, et al. Cardiovascular health: the importance of measuring patient-reported health status. Circulation. 2013;127(22):2233-49.

\section{Submit your next manuscript to BioMed Central and we will help you at every step:}

- We accept pre-submission inquiries

- Our selector tool helps you to find the most relevant journal

- We provide round the clock customer support

- Convenient online submission

- Thorough peer review

- Inclusion in PubMed and all major indexing services

- Maximum visibility for your research

Submit your manuscript at www.biomedcentral.com/submit

) Biomed Central 